\title{
Interferometer-Type Structures for Guided Atoms
}

\author{
R. Dumke, T. Müther, M. Volk, W. Ertmer, and G. Birk1* \\ Institut für Quantenoptik, Universität Hannover, Welfengarten 1, D-30167 Hannover, Germany
}

(Received 12 July 2002; published 7 November 2002)

\begin{abstract}
We experimentally demonstrate interferometer-type guiding structures for neutral atoms based on dipole potentials created by microfabricated optical systems. As a central element we use an array of atom waveguides being formed by focusing a red-detuned laser beam with an array of cylindrical microlenses. Combining two of these arrays, we realize X-shaped beam splitters and more complex systems like the geometries for Mach-Zehnder and Michelson-type interferometers for atoms.
\end{abstract}

DOI: $10.1103 /$ PhysRevLett.89.220402

PACS numbers: 03.75.Dg, 03.67.-a, 32.80.Pj, 42.50.-p

The investigation and exploitation of the wave properties of atomic matter is of great interest for fundamental as well as applied research and therefore constitutes one of the most active areas of research in atomic physics and quantum optics. Of special interest is the field of atom interferometry [1]. In comparison to optical interferometers, atom interferometers have the potential of being several orders of magnitude more sensitive for some applications or of giving access to classes of interferometric measurements not being possible with optical interferometry in principle. In the last decade, an impressive list of high-precision atom-interferometrical measurements of, e.g., fundamental constants, atomic properties, inertial forces, and rotations have been performed [2-10].

Because of the high intrinsic sensitivity, these interferometers have to be built in a robust way to be applicable under a wide range of environmental conditions. A new approach to meeting this challenge lies in the development of miniaturized and integrated atom optical setups based on microfabricated guiding structures. Using microfabricated current carrying wires, several configurations for atom guides [11-16] and beam splitters [17-19] also using Bose-Einstein condensates [20] have been realized. As an important goal remains the demonstration of a setup suitable as a guided-atom interferometer.

In this Letter we present the experimental implementation of atom guides, beam splitters, and structures for atom interferometers based on microfabricated optical elements as proposed in [21]. We demonstrate the guiding of neutral atoms along focal lines of arrays of microfabricated cylindrical lenses making use of optical dipole potentials. By superimposing two of these arrays under a variable relative angle, we realize $\mathrm{X}$-shaped beam splitters as well as interferometer-type configurations like Mach-Zehnder (Fig. 1) or Michelson-type structures. Because of the state selectivity of optical potentials, a state-selective guided-atom beam splitter could be demonstrated as well. Theoretical simulations of the wave dynamics predict the required coherence and interferometrical properties of these configurations [22] (see also [23]).
For the experiments presented here, we employ onedimensional arrays of cylindrical microlenses. Laser light sent through such a system forms a series of parallel focal lines above the lens array. Thus, for laser light detuned below an atomic resonance ("red-detuning") a one-dimensional array of atomic waveguides is formed [Fig. 2(a)] [24]. Atoms are confined in the two dimensions perpendicular to the lens axis but are free to propagate along the longitudinal axis for a homogeneous intensity distribution along the lens axis. Besides creating longitudinally flat potentials we can apply more complex intensity distributions, such as an intensity gradient for accelerating the atoms. A light field with a Gaussian intensity profile along the axis of the guide allows us to reverse the atom motion in the guide. This design flexibility gives the possibility to integrate atom mirrors with guiding structures in a direct fashion.

In our setup, the lens array consists of 12 lenses with a length of $5 \mathrm{~mm}$, manufactured in a fused sillica substrate. The center to center separation as well as the diameter of each lens is $0.4 \mathrm{~mm}$. The focal length is $2.21 \mathrm{~mm}$ giving a numerical aperture $\mathrm{NA}=0.09$. We image the focal plane of the microlens array onto the atoms to be guided with the help of two achromats

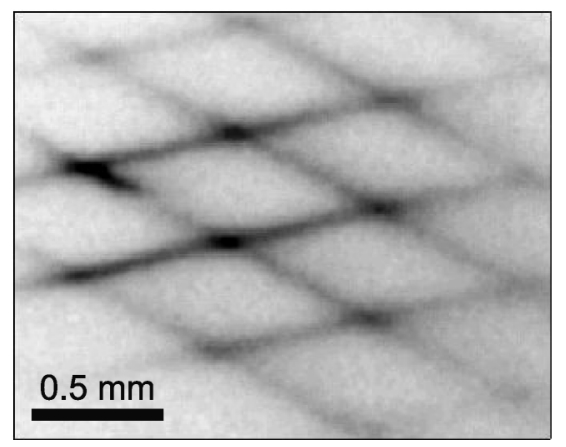

FIG. 1. Fluorescence image of rubidium atoms loaded from a MOT into a multiple-path Mach-Zehnder-type guiding structure created by the combined dipole potentials of two sets of microfabricated arrays of cylindrical lenses. 
$(\mathrm{NA}=0.08$, magnification $=1)$. The optical transfer of the trapping light has the advantage that we can place the micro-optical systems outside the vacuum chamber and thus can switch between and superimpose several microoptical elements easily [Fig. 2(b)]. The guiding of atoms close to the surface of these and of more complex integrated structures $[21,25]$ can be achieved in a straightforward fashion by putting the elements directly inside the vacuum chamber.

A typical experimental sequence starts by loading a single-dipole trap with about $10^{4}{ }^{85} \mathrm{Rb}$ atoms at a measured temperature of $20 \mu \mathrm{K}$ from a magneto-optical trap (MOT) (see [26] for details). After loading, the atoms are held in the single-dipole trap for $35 \mathrm{~ms}$, for untrapped atoms being able to leave the detection region. Then we transfer the atoms into the guiding structures by turning on the guiding and turning off the single-dipole trapping light. The loading efficiency is close to unity because the single-dipole trap is formed by illuminating a small part of the same cylindrical microlens, thus the reloading into the guide is achieved without significant loss in atom number or increase in temperature. Taking advantage of this, it should be possible to prepare the atoms by, e.g., Raman sideband cooling [27] in the ground state of the single-dipole trap and to reload them into the guiding structure adiabatically without changing the transversal vibrational state. The temporal evolution of the atom distribution in the guiding structures is observed via fluorescence imaging by a CCD camera with a spatial resolution of $14 \mu \mathrm{m}$ (rms spread). For detection, the guides are switched off and the atoms are illuminated by the MOT light for a period of $0.8 \mathrm{~ms}$.

The light used for atom guiding is derived from a single-frequency Ti:sapphire laser and has a typical power of $360 \mathrm{~mW}$ and a typical detuning $\Delta \lambda=1 \mathrm{~nm}$ $\left(\delta \nu=-500 \mathrm{GHz} \approx-5 \times 10^{5}\right.$ linewidths) "red" of the $5 S_{1 / 2} \rightarrow 5 P_{3 / 2}$ transition at $780 \mathrm{~nm}$. A typical potential depth for the waveguides is $450 \mu \mathrm{K}$. The radial (i.e., perpendicular to the laser beam direction) waist is $7 \mu \mathrm{m}\left(1 / e^{2}\right.$ radius of the intensity distribution) and the corresponding Rayleigh range is $200 \mu \mathrm{m}$. The calculated radial oscillation frequency is $9.6 \mathrm{kHz}$. From this and the measured temperature of $20 \mu \mathrm{K}$ we infer a mean occu-

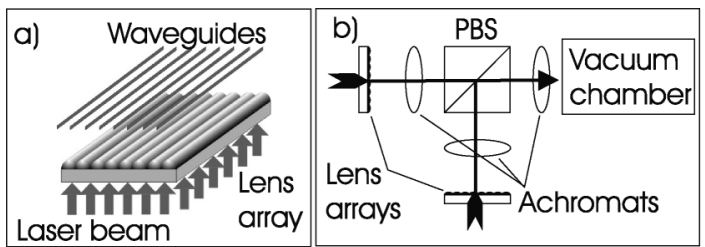

FIG. 2. (a) Array of atom waveguides formed by focusing a red-detuned laser beam with an array of cylindrical microlenses. (b) Schematic of the optical setup used for combining and transferring light fields: The focal planes of two lens arrays are reimaged by achromats and combined by a polarizing beam splitter (PBS). pation number of $\langle n\rangle \approx 40$ (radial direction) and a rms position spread of $\approx 1 \mu \mathrm{m}$ in radial direction and of $\approx 5 \mu \mathrm{m}$ along the laser beam direction. The rate for spontaneously scattered photons is $740 \mathrm{~s}^{-1}$ [28]. With this configuration we could observe the guiding of atoms over a typical distance of $2.5 \mathrm{~mm}$, limited by the accessible length of the lens array.

An important element for guided-atom interferometry is a beam splitter for atoms. Such a structure can be realized by combining the light fields of two microlens waveguides having one common focal plane but being oriented nonparallel to each other (see also [29]). The combination of the two waveguides results in an X-shaped beam splitter as shown in Fig. 3. Atoms are loaded from a single-dipole trap into the input port at left and are accelerated towards the intersection region by a gradient in the guiding potential. Here the paths split and the atoms are guided along both output ports. By rotating one lens array with respect to the other, the splitting angle can be chosen arbitrarily. For our experiments, it was set to $42^{\circ}$.

Interference effects in the combined light field at the intersections where the individual potentials add are avoided by polarizing the two light fields orthogonally [see Fig. 2(b)]. The transverse mode structure of the input port is identical to the one of the output ports with the potential being twice as deep at the intersection. Detailed calculations based on the parameters of our configuration show that the splitting process indeed is coherent for the guiding potential evolving adiabatically during the splitting process [22]. The highest possible degree of coherence can be achieved by compensating the doubling of the potential depth due to the overlap of the trapping potentials at the intersection by an optimized lens design [21] or by overlapping the intersection region with the repulsive potential of a blue-detuned laser beam [30].

We could demonstrate a variation in the splitting ratio over a wide range by changing the power ratio between the two guides forming the beam splitter (Fig. 4). The atom number in the two output ports is determined by taking line profiles similar to Fig. 3(b) and integrating the density distribution for each port. By choosing an appropriate power ratio, which depends on the velocity

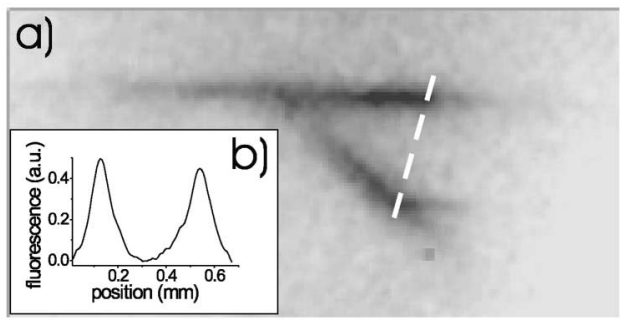

FIG. 3. Beam splitter for guided atoms: (a) Fluorescence image of atoms guided through a beam splitter based on two crossed cylindrical microlenses. (b) Line profile of the atom distribution along the dotted line of (a). 


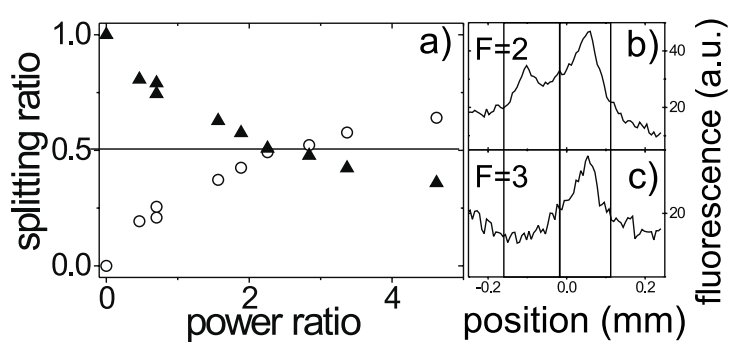

FIG. 4. Properties of different types of beam splitters: (a) The splitting ratio for atoms in a state-insensitive beam splitter is altered by varying the intensity ratio between the waveguides creating the beam splitter. (b) and (c) Line profiles for atoms after moving through a state-selective beam splitter: (b) Atoms in the state $5 S_{1 / 2}(F=2)$ evolve through the beam splitter unperturbed. The line profile shows atoms in both outputs. (c) Atoms in the state $5 S_{1 / 2}(F=3)$ can propagate only along one output.

of the atoms and the splitting angle, a splitting ratio of $50 / 50$ can be achieved.

So far, the splitting process was fully determined by the external degrees of freedom. Specific to optical guiding structures is the possibility to use the internal atomic structure for the splitting process, similar to RamseyBordé [2,8] and Raman interferometers [3] for free atoms. For guided atoms, state-selective splitting can be achieved, for example, by applying an additional stateselective optical potential in a small section of one output shortly behind the beam splitter of Fig. 3. We implemented this for ${ }^{85} \mathrm{Rb}$ by employing an additional laser field with a red-detuning of $\delta \nu_{2}=-1020 \mathrm{MHz}(-170$ linewidths) for atoms in the $5 S_{1 / 2}(F=2)$ hyperfine ground state. The same laser field is blue-detuned for atoms in the $5 S_{1 / 2}(F=3)$ hyperfine ground state with a detuning of $\delta \nu_{3}=2020 \mathrm{MHz}$ (340 linewidths) [31]. State-selective splitting for a guided-atom beam splitter is shown in Figs. 4(b) and 4(c). For atoms in the $5 S_{1 / 2}(F=2)$ state, after the intersection there is one unperturbed output and one with an additional potential well (simply acting as a phase shifter). Therefore the atoms propagate along both outputs of the splitter [Fig. 4(b)]. Atoms initially prepared in the $5 S_{1 / 2}(F=$ 3) state cannot propagate along the output with the additional potential barrier caused by the blue-detuned light field [Fig. 4(c)]. A foreseeable application of this technique will lead to the preparation of atoms in a superposition of the two hyperfine ground states prior to or right at the beam splitter, so that the splitting process will be determined by the internal superposition state. With such a system it should be possible to create robust coherent beam splitters for guided atoms based on their internal states.

As being the central goal of this work, we designed and experimentally demonstrated the structures for two integrated interferometer-type configurations for guided atoms: (i) Mach-Zender-type (Figs. 1 and 5) and (ii) Michelson-type (Fig. 6) structures.

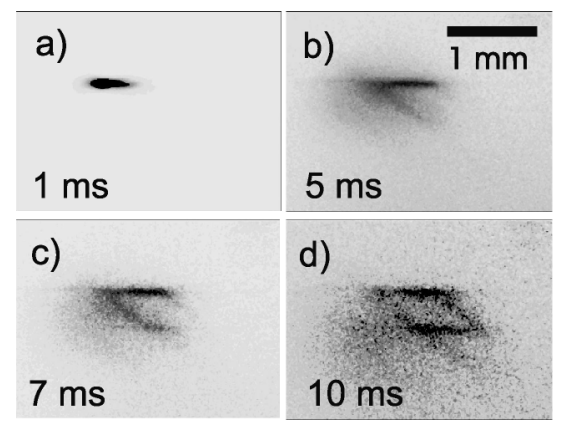

FIG. 5. Temporal evolution of atoms propagating through a Mach-Zehnder-type interferometer structure: Loaded from a single dipole trap (a) the atoms are split by the input beam splitter (b) and propagate along two different paths to the output beam splitter (c),(d).

By combining two arrays of waveguides we create multiple X-shaped beam splitters. A set of four of these beam splitters represents the basic Mach-Zehnder-type structure (Fig. 5) with the option of using the additional input and output ports for creating more complex multipath guided-atom interferometers (Fig. 1), e.g., for increased sensitivity [32]. The basic configuration acts as a closed loop structure with a finite enclosed area as required for a Sagnac-type interferometer. Figure 5 shows the propagation of atoms through a Mach-Zehnder-type structure for guided atoms. We load one of the input ports with atoms from the single-dipole trap (a). Atoms propagate to the first beam splitter and split into two paths (b). At the next intersections these split into a total of four paths (c). Two of the paths recombine at the the fourth intersection (d). This Mach-Zehnder-type structure has an enclosed area of $0.3 \mathrm{~mm}^{2}$ with a total required array below $1 \mathrm{~mm}^{2}$ including the loading and detection regions. It presents the first experimental demonstration of a closed structure suitable for atom interferometry based on atom guides. In numerical simulations, it could be demonstrated that for typical experimental conditions coherent splitting of atom-waves and matter-wave interference at the outputs can be achieved [22]. As an important result, a variation in the relative phase between the two paths of a Mach-Zehnder-type structure resulted in a complementary periodic variation of atom number in

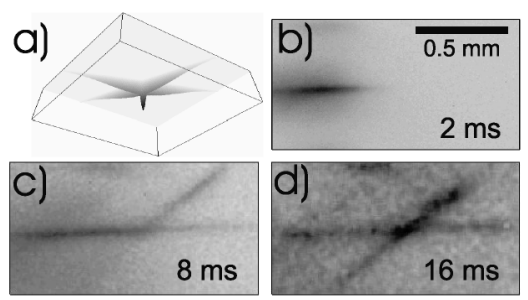

FIG. 6. Configuration of a Michelson-type interferometer: (a) Structure based on two crossed waveguides with a Gaussian intensity profile along the longitudinal direction. (b)-(d) Propagaton of atoms in the Michelson-type structure loaded from a single dipole trap. 
the two final output ports, thus clearly predicting the existence of interference fringes.

We also demonstrated a Michelson-type interferometer structure (Fig. 6) by crossing two micro-optical guides in our "standard" beam splitter configuration, each guide now having a Gaussian intensity profile along the longitudinal direction centered at the intersection [Fig. 6(a)]. We load atoms in one input close to the edge of the potential well. The atoms are accelerated towards the beam splitter [Fig. 6(b)]. They split into two paths and are slowed down by the positive gradient of the potential [Fig. 6(c)]. They start moving backwards towards the intersection. At the intersection they split again with part of the atoms now also moving along the output port of the Michelson-type structure [Fig. 6(d)]. The oscillation along the longitudinal direction is significantly slower than in the transverse directions. Thus, an adiabatic propagation through the structure is easily achievable.

In this Letter we have presented the first experimental demonstration of structures for guided-atom interferometers based on microfabricated elements. For this purpose, guides and beam splitters based on micro-optical elements have been employed. Theoretical investigations predict the feasibility of coherent beam splitting and the possibility of achieving matter-wave interference for guided atoms. However, several modifications to our apparatus, such as improved atomic state preparation, detection resolution, and detection efficiency need to be implemented prior to a demonstration of coherence. Interferometer structures optimized for coherence and fringe contrast are currently being developed and the demonstration of an interference experiment will be attempted under optimized experimental conditions. The micro-optical systems investigated here are first realizations of a broad class of configuration being accessible due to the high flexibility in the manufacturing process of these elements [21]. Applications hugely benefit from the many inherent advantages of integrated systems, such as stability and scalability. The configurations described here present a major step towards miniaturization of atom-interferometrical devices and promote the wide practical applicability of various classes of sensors based on atom interferometry.

We thank F. Buchkremer, H. Kreutzmann, M. Lewenstein, and A. Sanpera for many stimulating discussions. This work is supported by the SFB 407 and the SPP Quanteninformationsverarbeitung of the Deutsche Forschungsgemeinschaft and by the project ACQUIRE (IST-1999-11055) of the European Commission.

*Author to whom correspondence should be addressed.

[1] Atom Interferometry, edited by P. R. Berman (Academic Press, San Diego, 1997), and references therein.
[2] U. Sterr et al., Appl. Phys. B 54, 341 (1992).

[3] D. S. Weiss, B. C. Young, and S. Chu, Phys. Rev. Lett. 70, 2706 (1993).

[4] C. R. Ekstrom et al., Phys. Rev. A 51, 3883 (1995).

[5] K. Gibble and S. Chu, Phys. Rev. Lett. 70, 1771 (1993); C. Fertig and K. Gibble, Phys. Rev. Lett. 85, 1622 (2000).

[6] S. Ghezali et al., Europhys. Lett. 36, 25 (1996); Y. Sortais et al., Phys. Rev. Lett. 85, 3117 (2000).

[7] M. Kasevich and S. Chu, Phys. Rev. Lett. 67, 181 (1991); M. J. Snadden et al., Phys. Rev. Lett. 81, 971 (1998); A. Peters, K. Y. Chung, and S. Chu, Nature (London) 400, 849 (1999).

[8] F. Riehle et al., Phys. Rev. Lett. 67, 177 (1991).

[9] A. Lenef et al., Phys. Rev. Lett. 78, 760 (1997).

[10] T. L. Gustavson, P. Bouyer, and M. A. Kasevich, Phys. Rev. Lett. 78, 2046 (1997); T. L. Gustavson et al., Classical Quantum Gravity 17, 2385 (2000).

[11] D. Müller et al., Phys. Rev. Lett. 83, 5194 (1999).

[12] N. H. Dekker et al., Phys. Rev. Lett. 84, 1124 (2000).

[13] M. Key et al., Phys. Rev. Lett. 84, 1371 (2000).

[14] R. Folman et al., Phys. Rev. Lett. 84, 4749 (2000).

[15] P. Engels, W. Ertmer, and K. Sengstock, Opt. Commun. 204, 185 (2002).

[16] A.I. Sidorov et al., Acta Phys. Pol. B 33, 2137 (2002).

[17] D. Cassettari et al., Phys. Rev. Lett. 85, 5483 (2000).

[18] D. Müller et al., Opt. Lett. 25, 1382 (2000); D. Müller et al., Phys. Rev. A 63, 041602(R) (2001).

[19] W. Hänsel et al., Phys. Rev. Lett. 86, 608 (2001).

[20] W. Hänsel et al., Nature (London) 413, 498 (2001); H. Ott et al., Phys. Rev. Lett. 87, 230401 (2001); A. E. Leanhardt et al., Phys. Rev. Lett. 89, 040401 (2002); see also forthcoming publications by the groups of J. Schmiedmayer, E. Hinds, and E. A. Cornell.

[21] G. Birkl et al., Opt. Commun. 191, 67 (2001).

[22] H. Kreutzmann et al. (to be published).

[23] E. Andersson et al., Phys. Rev. A 59, 3841 (1999); E. Andersson et al., Phys. Rev. Lett. 88, 100401 (2002).

[24] The high reproducibility of the fabrication process suppresses variations between individual lenslets leaving the temporal and spatial variations of the laser light as the main source of fluctuations in the guiding potentials.

[25] J. P. Burke et al., Phys. Rev. A 65, 043411 (2002).

[26] R. Dumke et al., Phys. Rev. Lett. 89, 097903 (2002).

[27] S. E. Hamann et al., Phys. Rev. Lett. 80, 4149 (1998); V. Vuletic et al., Phys. Rev. Lett. 81, 5768 (1998); H. Perrin et al., Europhys. Lett. 42, 395 (1998).

[28] Lens arrays with optimized numerical aperture will give further improved waveguide parameters (see [21]).

[29] O. Houde et al., Phys. Rev. Lett. 85, 5543 (2000).

[30] The calculations of [22] show that significant coherence is still maintained during the splitting process with a mismatch in the potential compensation of up to $20 \%$.

[31] Other implementations of a state-selective beam splitter can be based on the polarization dependence of the optical potentials for different internal atomic states.

[32] M. Weitz, T. Heupel, and T.W. Hänsch, Phys. Rev. Lett. 77, 2356 (1996); H. Hinderthür et al., Phys. Rev. A 59, 2216 (1999). 